\title{
Amélioration de la qualité de la formation à l'aide de la certification académique et professionnelle
}

\author{
S. CHOUGDALI ${ }^{1}$, A. ROUDANE ${ }^{1}$, A. REBBANI ${ }^{1}$, K. MANSOURI ${ }^{1}$, M. YOUSSFI ${ }^{1}$ \\ ${ }^{1}$ Laboratoire : Signaux, Systèmes Distribués et Intelligence Artificielle (SSDIA) \\ ENSET, Université Hassan II, Mohammedia Casablanca \\ Emails: $\underline{\text { s.chougdali@gmx.fr, asmaaroudane@gmail.com, a.rebbani@gmail.com, }}$ \\ khmansouri@hotmail.com, med@youssfi.net
}

\section{Résumé}

La certification académique ou professionnelle est un ensemble de processus et de normes qui permettent de mesurer le niveau de compétence à un moment donné.

Les entreprises modernes n'exigent plus que des diplômes classiques délivrés traditionnellement par les universités ou les écoles, mais exigent des certifications permettant de valider des acquis précis dans des domaines pointus vérifiés dans un cadre international.

Dans cet article, nous revenons, brièvement, sur la définition de la formation et celle de la certification. Nous établissons le lien entre les deux ainsi que le rôle et les avantages de la certification pour une bonne formation. Nous étudions ensuite la différence entre une certification professionnelle et une certification académique. Nous poursuivons par la présentation de quelques exemples de certifications dans le domaine des TIC, ainsi que l'impact de ces certifications sur la réussite des lauréats dans la vie professionnelle.

Nous présenterons enfin l'expérience de l'École Normale Supérieure d'Enseignement Technique (ENSET) de Mohammedia, dans le domaine de la certification académique.

Mots clés : Formation- Connaissances - Compétences - Évaluation - Certification Certifications académiques - Certifications professionnelles.

\section{Introduction générale}

L'évaluation de la qualité dans les systèmes d'éducation et de formation est un phénomène en pleine expansion, vu que c'est un facteur critique, qui contribue et mesure le développement des pays, d'où un très grand intérêt doit être réservé à ces systèmes.

Plusieurs réformes ont été appliqués afin d'améliorer la qualité de l'enseignement supérieur, notamment l'adoption de plusieurs modes de certifications académiques et professionnelles. Ces réformes ont pour finalité de former des lauréats capables de satisfaire les besoins évolutifs des entreprises, mais aussi la création de profils ayant la possibilité de mettre facilement en pratique les connaissances théoriques acquises à l'école ou à l'université.

Ces nouveaux outils qui permettent de renforcer l'enseignement traditionnel et le rendre plus efficace, nécessitent une étude très approfondie d'où l'intérêt de traiter, dans cet article, les apports de la certification, comme outil de perfectionnement, sur la qualité de la formation.

\section{Généralités sur la formation et la certification}

\subsection{La formation}

La formation est un processus à finalités multiples et imbriquées. Elle s'adresse à des individus et s'évalue par une mesure de l'adéquation entre les comportements exhibés par les apprenants et les situations proposées par le formateur. Elle ne peut donc se réduire à la simple transmission d'un programme d'enseignement. La technique doit prendre en compte les caractéristiques comportementales et cognitives des apprenants. [1]

La formation initiale est une formation très structurée par des programmes rigides qui durent généralement plusieurs années et qui demandent souvent une participation à temps complet. 
Ce type de formation traditionnelle est dispensé par les institutions d'enseignement ordinaires comme les écoles et les universités, et est conçue pour une population définie a priori (élèves, étudiants,...). Il s'agit d'un processus de scolarisation donnant accès à une reconnaissance sociale et à un diplôme correspondant au niveau de scolarité atteint. [2]

L'évolution des besoins de l'entreprise en termes de compétences professionnelles spécialisées a donné naissance à la formation professionnelle et continue préparant à la pratique d'un métier. Il serait donc plus raisonnable de construire la formation à partir d'une analyse réaliste des pratiques de référence. C'est-à-dire qu'il faut proposer des formations en identifiant les compétences cibles en rapport avec les besoins de l'entreprise.

L'objectif principal de la formation que ça soit initiale, professionnelle ou continue est de mener l'apprenant, généralement un étudiant ou un employé, vers la professionnalisation. Ceci se réalise à travers le développement de ses capacités d'analyse, de décision et de prise en charge de toute situation rencontrée pendant l'exercice d'un travail. [11, 12, 13, 14]

Le développement des technologies de l'information et de la communication et l'introduction de ces technologies numériques dans l'environnement universitaire a changé les méthodes de formation. Aujourd'hui les universités sont appelées à trouver d'une façon créative et efficace des solutions s'adaptant avec les flux importants des étudiants tout en assurant une formation de qualité et un environnement favorable pour un bon apprentissage. C'est pour cela que la mise en place des plateformes E-learning et des espaces numériques de travail pour une formation à distance est considérée comme la tendance. Ainsi l'utilisation de l'Internet pour la diffusion d'un contenu de formation, appelée MOOC (Massive Open Online Course), est considérée comme exemple de formation ouvert et à distance. Elle permet à un enseignant de diffuser ses cours sur une plateforme. Plusieurs apprenants dispersés géographiquement peuvent suivre ces cours en ligne et en même temps. Cette technique a été adoptée dernièrement, pour la première fois, en Afrique, par l'université Cadi Ayyad à Marrakech.

\subsection{La Certification}

Pour suivre les évolutions du système éducatif et du système d'emploi et afin de garder le lien, devenu de plus en plus fort, entre les deux et ayant un impact positif sur le taux de recrutement, la préparation des certifications en parallèle avec le suivi d'une formation, que ça soit initiale, professionnelle ou continue est devenu incontournable. [7, 9, 10]

Le terme certification est générique, il s'applique à un grand nombre d'objets et d'actes officiels ou non. Ne seront considérées ici que les certifications concernant le processus de vérification d'une maîtrise professionnelle (Sous l'angle des personnes) et son résultat. Ne sont pas considérées ici les certifications s'appliquant aux entreprises. (Par exemple de type ISO).

La certification est donc un processus par lequel l'organisme certificateur donne une assurance écrite sous forme d'un certificat, d'un diplôme ou d'une attestation à une personne disposant d'un certain niveau de compétences et de connaissances conformes à des exigences spécifiées dans le référentiel de certification.

La certification a pour rôle principal de valider la reconnaissance d'un savoir-faire. C'est un acte volontaire qui ne peut en aucun cas remplacer la formation académique qui reste un processus d'enseignement et d'apprentissage ayant un rôle formateur. Par contre, la certification mesure le niveau de compétence d'un individu à un moment donné.

\subsection{Différence entre certification professionnelle et académique}

L'analyse du secteur très large des certifications permet de les classer en deux catégories :

La certification professionnelle qui permet d'attester la capacité à réaliser des activités professionnelles dans le cadre de plusieurs situations de travail, à des degrés de responsabilités définis dans un référentiel. Cette certification est généralement crée par les 
professionnels de la formation pour développer des compétences en liaison avec un métier précis. Elle est construite pour répondre à des besoins spécifiques sur la base d'activités et de compétences propres à chaque métier. Elle est délivrée par des organismes certificateurs reconnus et présente une valeur ajoutée qui peut bien distinguer l'individu au moment du recrutement. [5]

La certification académique est traduite par un titre délivré par une entreprise de formation, un institut, une école ou une université attestant que l'apprenant a suivi une formation selon un programme bien déterminé et a passé les évaluations liées à ce parcours.

\subsection{Impact de la certification sur la qualité de formation et la réussite des lauréats dans la vie professionnelle $[3,14]$}

Pour étudier le rôle de la certification dans la réussite des apprenants, plusieurs approches peuvent être considérées. La certification est un signal de productivité, un filtre de sélection, un réducteur d'incertitude, un élément de confiance, un outil de validation de la qualité de la formation, un compliment de la formation, un investissement de forme, un outil de gestion de ressources humaines ou un outil de sécurité du parcours professionnel.

La certification consiste en une véritable reconnaissance technique. Elle permet au certifié de se positionner comme spécialiste dans un domaine précis à côté de la formation. Elle est considérée comme valeur ajoutée dans son $\mathrm{CV}$. Le certifié peut alors bénéficier d'une grande facilité dans la négociation de son salaire et des meilleures opportunités professionnelles.

La certification garantit aussi, un niveau d'expertise sur un produit ou une technologie donnée. C'est d'autant plus important que de nombreuses entreprises exigent dans leur appel d'offres que les intervenants détiennent la certification à tel ou tel outil. Parfois le certificat est reconnu à l'échelle internationale, ce qui permet au certifié de décrocher facilement une embauche respectueuse à l'étranger.

\subsection{Démarche d'une certification}

Pour accéder à la certification, plusieurs façons peuvent être considérées :

- À l'issu d'une formation, comme étant un étudiant, un salarié ou un chercheur d'emploi.

- Comme candidat libre. Dans ce cas, la certification est considérée comme une action volontaire par un individu qui a de l'intérêt d'enrichir son $\mathrm{CV}$.

- Parfois via la VAE (Validation des Acquis de l'Expérience) c'est une manière pour obtenir un niveau d'une certification professionnelle en s'appuyant sur l'expérience professionnelle de l'individu à condition qu'elle soit en rapport direct avec le contenu de la certification.

La démarche d'une certification peut s'articuler essentiellement sur 5 étapes :

- Étape 1 : l'individu doit choisir la certification qui correspond à son profil et dépose sa candidature auprès de l'organisme certificateur.

- Étape 2 : l'organisme certificateur traite la candidature de l'individu et décide si le candidat est apte à passer la certification demandée ou non.

- Étape 3 : l'individu participe à une ou plusieurs formations s'il est nécessaire.

- Étape 4 : l'individu passe l'examen soit en ligne ou aux locaux de l'organisme certificateur.

- Étape 5 : suite aux résultats de l'examen et s'ils sont conformes aux référentiels de la certification, le candidat devient certifié.

\subsection{Quelques exemples de certifications dans le domaine des TIC.}

Plusieurs études montrent que les systèmes éducatifs ne préparent pas correctement les étudiants à l'économie digitale. D'où la nécessité de mettre en place des points de repère s'appuyant sur des certifications professionnelles. 


\section{a. Réseaux informatiques (CISCO systems, ...)}

Les certifications Cisco, reconnues comme la norme industrielle en matière de conception et d'assistance réseau, garantissent de hauts niveaux de spécialisation et de crédibilité. Depuis les technologies élémentaires de mise en réseau jusqu'aux domaines techniques plus spécifiques et plus évolués comme la sécurité, le sans fil, les réseaux de diffusion de contenu et la téléphonie IP. Les certifications ciblées Cisco valident les connaissances, garantissent les compétences professionnelles et multiplient les opportunités de carrière et d'avancement. Avec des employés certifiés par Cisco, l'entreprise peut tirer le meilleur parti de son investissement de réseau en minimisant les temps de panne, en renforçant la productivité de ses collaborateurs et en améliorant la satisfaction de ses clients.

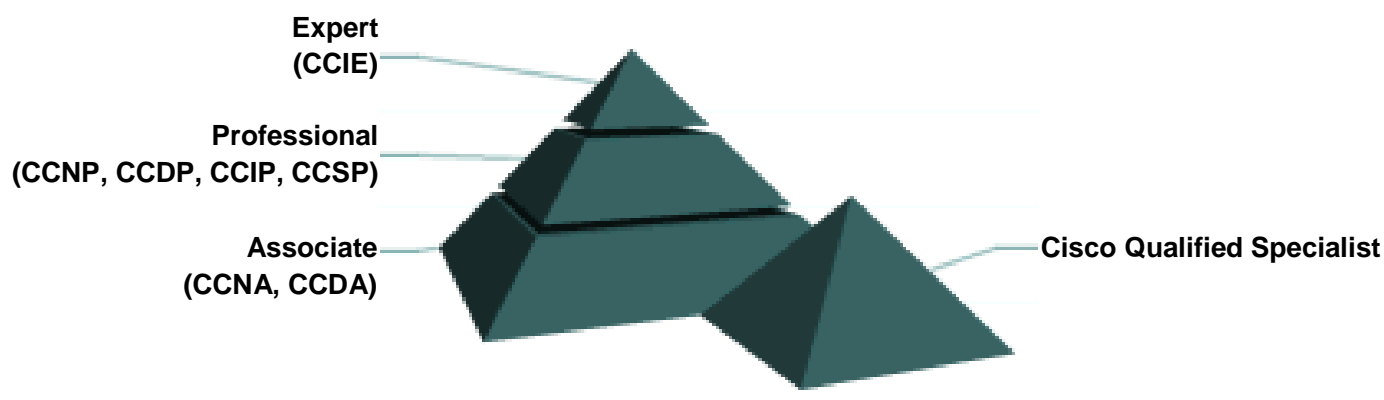

Figure 1 : Types de certifications Cisco

Cisco propose trois certifications générales représentant des niveaux croissants de compétences : Associate, Professionnal et Expert. Les différentes passerelles entre ces niveaux correspondent aux différents besoins de carrière. Un grand nombre de certifications Cisco Qualified Specialist sont également proposées et garantissent la maîtrise des connaissances dans des technologies, des solutions ou des fonctions spécifiques. [17]

\section{b. Outils Microsoft (Systèmes, Développement, Office, ...)}

Les certifications Microsoft sont une valeur ajoutée qui valorise l'expérience des apprenants concernés de manière générale à mieux utiliser les outils informatiques dans leurs futurs métiers et cela en permettant d'augmenter leur employabilité.

Microsoft offre plusieurs certifications concernant ses produits comme: MS Office, SqlServer, Windows Server 2003, 2008, et bien d'autres.

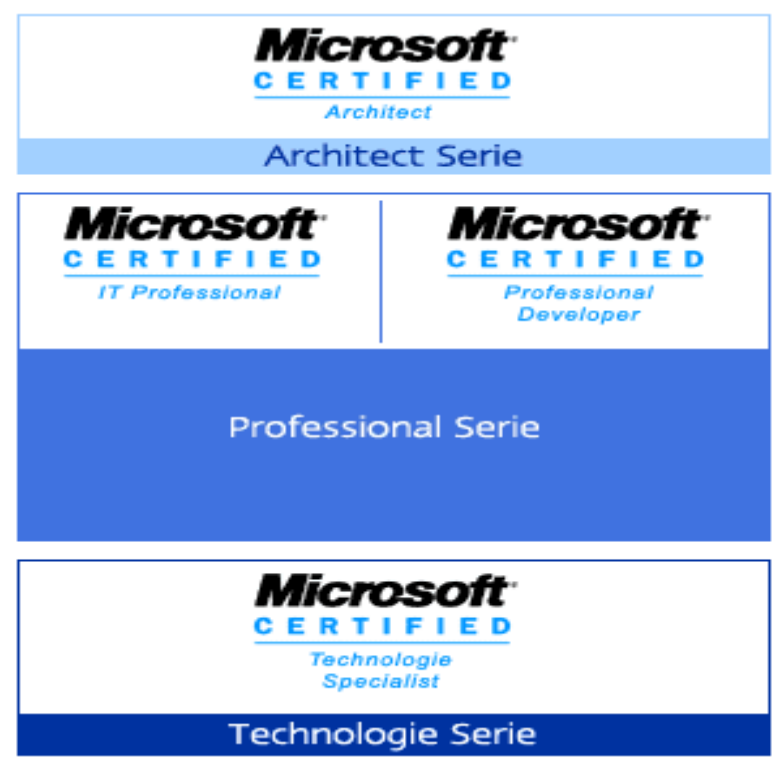

Figure 2 : Arborescence parallèle des certifications Microsoft 
Exemples de certifications Microsoft : [18]

- Certification MOS : Certification sur l'ensemble de produits Office, en version 2003 et 2010. Trois niveaux : MOS Specialist, MOS Expert et MOS Master

- Certification MTA : Certification pour valider les connaissances informatiques de base et notamment le développement de logiciels et l'administration de base de données. Les examens disponibles sont : développement logiciel, développement web, développement Windows, administration de Windows Server, le réseau, administration de Base De Données, administration Sécurité.

\section{c. Méthodes de gouvernance des systèmes d'information (SI)}

- COBIT : Le référentiel COBIT, Control objectives for information and technology proposé par l'ISACA, est un cadre de référence orienté processus de l'audit et de la maîtrise du SI. COBIT propose les "meilleures pratiques" pour gérer efficacement l'alignement des technologies dans une logique de maîtrise des risques et des investissements. [15]

- ITIL : Information Technology Infrastructure Library est le fruit d'un travail conduit par l'Office Public du Commerce Britannique (OGC Office of Government Commerce). Il s'agit d'une sélection de bonnes pratiques orientées clients afin d'assurer une gestion efficace, risques et qualité, des services informatiques. [15]

- CMMI Capability Maturity Model Integration est un modèle d'évaluation orienté processus de la capacité à atteindre les objectifs en matière de réalisation informatique. Fondé sur un référentiel de bonnes pratiques, il s'inscrit dans une logique d'amélioration continue. [15]

\section{d. Développement informatique (JAVA, Dot Net, etc...)}

Sun MicroSystems propose différentes certifications Java :

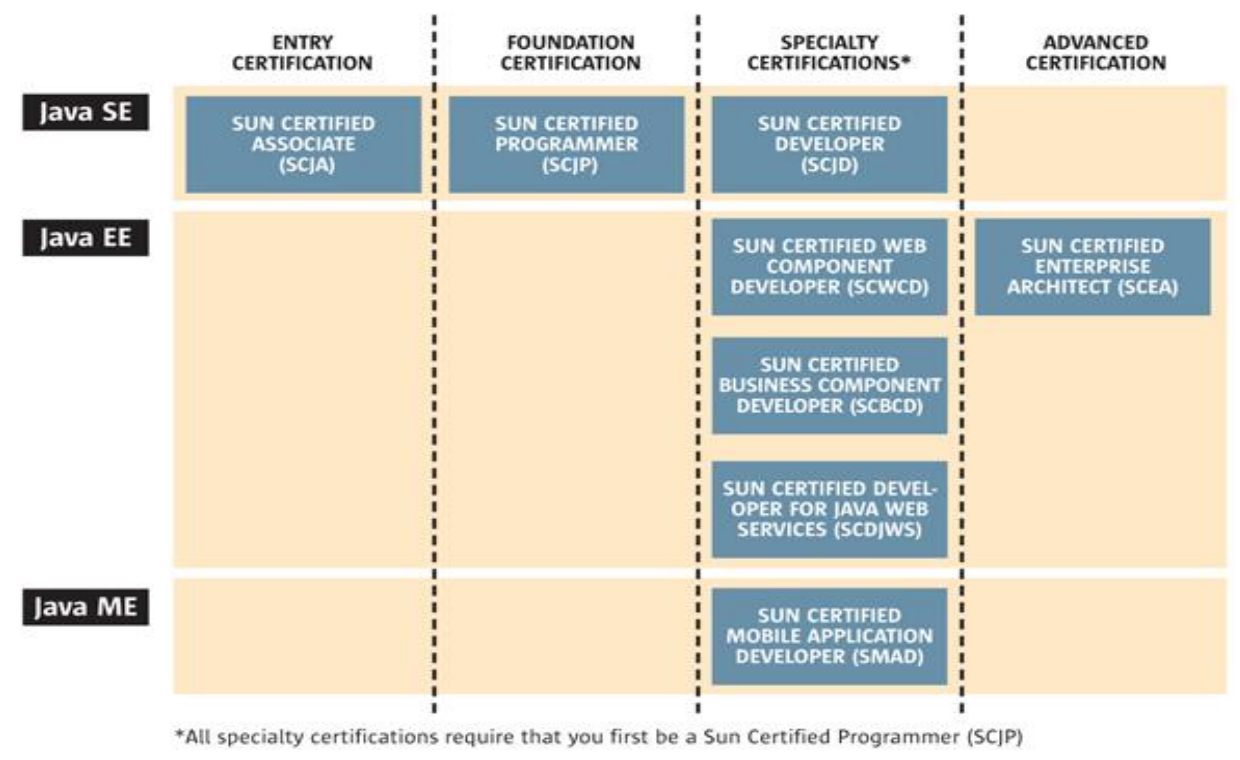

Figure 3 : Les certifications JAVA proposées par Sun MicroSystems

\section{Exemple de la certification SMAD : (Sun Micro Application Developer)}

La certification SMAD concerne les personnes qui développent des applications sous la plateforme Java ME. CX310-110 SMAD pour la plateforme Java ME. Pour l'examen CX310110, il faudra répondre à 68 questions en 150 minutes et avoir au moins $55 \%$ pour réussir.

\section{e. Gestion des bases de données (SQL, Oracle, etc...)}

Les analystes de l'industrie considèrent que les Certifications Oracle sont les plus recherchés pour valoriser l'expertise IT. La Certification OCP DBA était le seul certificat classé dans le 
Top 10 des certificats reconnus par CRN.com 2002 [16]. Les professionnels IT peuvent se différencier de leurs pairs et de la concurrence et sécuriser leur emploi en devenant certifiés.

Les certifications Oracle sont désignées par des codes comme : 1Z0-xxx (Ex : 1Z0-007, 1Z0147 etc...). Les numéros changent en fonction de la version de la base sur laquelle on est certifiés. Par exemple, la DBA I 10G est désignée par 1Z0-042 tandis que la DBA I 11G l'est par 1Z0-052. Lorsque l'on obtient une, elle est valable à vie. Cependant, si on veut nous certifier sur la nouvelle version d'Oracle Database $11 \mathrm{G}$ et qu'on a déjà la même certification $10 \mathrm{G}$, on doit repasser une certification de mise à niveau.

La plupart des certifications sont organisées en deux grandes catégories : développement PL/SQL et Administration/Maintenance d'une base Oracle. Chacune des catégories permettra d'obtenir divers titres comme Oracle Certified Associate (OCA), Oracle Certified Professional (OCP) ou Oracle Certified Master (OCM).

\section{Expérience de l'ENSET Mohammedia dans le domaine de certifications}

Pour permettre à ses lauréats de se mesurer à des référentiels internationaux et les doter d'outils performants consolidant leurs formations et facilitant leurs insertions dans le monde socio économiques, l'ENSET de Mohammedia a mis en place, en collaboration avec plusieurs organismes extérieurs spécialisés, un certain nombre de certifications académiques dans le domaine des nouvelles technologies de l'information et de la communication en formation initiale et/ou en formation continue.

C'est ainsi qu'elle a fait bénéficier ses lauréats :

- De la certification Cisco en mettant en place une académie locale gérée par deux enseignants spécialistes,

- De la certification Microsoft et Systèmes en collaboration avec la société IT Learning Accademy et LSI Maroc supervisées par trois enseignants spécialistes.

\subsection{Réseaux informatiques (CISCO systems, ...) [17]}

Dans ce domaine, l'ENSET a préparé ses étudiants à la certification CCNA (Cisco Certified Network Assciate Level), qui offre aux étudiants un enseignement générique sur les technologies réseaux.

Les étudiants qui bénéficient de cette certification sont ceux inscris dans une licence professionnelle et master spécialisé en formation initiale et dans deux licences d'université en formation continue.

Cette certification s'est déroulée de la manière suivante : Inscription des étudiants sur le site Netacad, Activation des cours puis Evaluation en ligne.

\subsection{Outils Microsoft (systèmes, ...)}

Dans ce domaine, l'ENSET a préparé ses étudiants aux certifications suivantes : 2275 Windows 2003 Server, 2276 Windows 2003 Server et 2285 Windows XP SP2.

Les étudiants qui ont bénéficié de cette certification sont ceux inscris dans deux licences d'université en formation continue.

Cette certification s'est déroulée de la manière suivante: Définir et Adapter le projet professionnel par rapport à la filière et au groupe, réaliser une étude de cas, faire l'étude de l'existant (Windows 2003 server), proposer une solution Migration Windows 2003 vers Windows 2008 Server, gérer, planifier et réaliser le déploiement du projet.

D'après l'enseignant responsable, les projets étudiés dans le cadre de ces certifications sont des cas réels qui ont été implémentés durant sa carrière professionnelle au Maroc et dans 
d'autres pays de l'Afrique au sein de plusieurs grands organismes tels que les banques, les assurances, les grandes surfaces et d'autres grands groupes très connus mondialement.

\subsection{Développements informatiques (Dot Net, ASP, etc...)}

Dans ce domaine, l'ENSET a préparé ses étudiants à la certification suivante :

- Course 70010 : Creating and Adding Functionality to a Microsoft ASP.NET Web Form

- Course 70015: Managing State, Configuring, Deploying an ASP.NET Web Application

- Course 70009: Introduction To Microsoft ASP.NET Web Applications

- Course 70011: Implementing Master page And user Controls Validating user input

- Course 70001 : Web Application Architecture and Design

- Course 70005 : Web Form Data Management Application

Les étudiants qui ont bénéficié de cette certification sont ceux inscris dans deux licences d'université en formation continue.

Les cours et ateliers se sont bien déroulés. Cependant, il faut noter que la préparation aux modules de certificat demande des efforts particuliers de la part du formateur et un niveau intéressant en anglais des candidats à certifier, car tous les examens sont en langue anglaise.

\subsection{Avis des enseignants qui ont assuré ces certifications}

\section{a. Certification en réseaux informatiques}

D'après l'enseignant responsable :

- L'impact de cette certification sur la formation des étudiants est immédiat, car les cours sont sanctionnés par des évaluations en ligne qui donnent à l'étudiant des informations immédiates sur ses travaux.

- L'impact de cette certification sur l'embauche des étudiants : de nombreux diplômés poursuivent des carrières prospères dans le domaine des technologies de l'information et de la communication dans une multitude de secteurs. D'autres ont mis à profit l'esprit d'entreprise et les connaissances acquises par le biais du programme Networking Academy pour lancer leur propre société et créer de nouveaux emplois.

- Le taux de réussite des étudiants dans cette certification est de 80\%.

- Les étudiants sont très satisfaits. Cependant ils réclament plus de volume horaire et l'installation de nouveau labs (équipements) actualisés.

- Les principaux problèmes rencontrés sont : disponibilité et débit de la connexion Internet, effectifs des étudiants pendant les TP et disponibilité des ordinateurs (machines en pannes). Pour essayer de remédier à ces problèmes, les étudiants ramènent leur propre machine et des connexions Internet modem $3 \mathrm{G}$

- Les conseils donnés aux étudiants sont de donner de l'importance à la certification académique et préparer puis passer les certifications professionnelles.

- Les conseils donnés aux enseignants qui désirent certifier des étudiants sont d'abord de se certifier soit même et de suivre l'actualité de la certification académique.

- Les recommandations du responsable sont de mettre en place plus de certifications académiques et dans différents domaines, sensibiliser le corps professoral à l'intérêt des certifications et faire une étude sur l'impact de la certification sur l'embauche des certifiés.

\section{b. Certification en Systèmes}

- D'après l'expérience du responsable, comme Consultant Informatique, les certifications ajoutent de la valeur à chaque bénéficiaire. Avec des certifications, il a plus de chances à être accepté dans un entretien de recrutement et à s'insérer facilement dans les entreprises. 
- Le taux de réussite des étudiants dans chaque certification peut atteindre les $100 \%$, tout dépend des groupes, des individus et de l'intérêt qu'ils portent à ces certifications.

- Les étudiants sont très satisfaits grâce aux projets métier très pratiques donnés par les enseignants. En fait les étudiants ont toujours demandé plus de projets à travailler.

- La remarque générale soulevée par les étudiants est le démarrage tardif des certifications par rapport à celui de la formation. Ils réclament aussi des réunions régulières chaque mois avec le responsable de la formation et celui de la certification pour plus de régulation.

- Les principaux problèmes rencontrés sont : la différence de niveau des étudiants qui influence l'avancement du travail de certification, le manque d'équipement adéquat pour la certification, la connexion à Internet doit être via des câbles au lieu de la technologie Wifi qui cause beaucoup de problèmes pendant le passage des certificats et le nombre d'étudiants à certifier ne doit pas dépasser 24 par groupe. L'enseignant responsable propose l'utilisation des Modems 3G+ pour chaque étudiant.

- Le conseil donné aux étudiants est que dans le milieu professionnel, on doit toujours essayer de rester à jour et suivre l'évolution technologique en passant plus de certifications récentes.

- Le conseil donné aux enseignants désirant certifier des étudiants est qu'ils doivent adapter leurs cours et Labs métier vers tout ce qui est plus professionnel afin d'aider les étudiants à bien se préparer au passage des certifications pour mieux se présenter au monde du Travail.

- La recommandation du responsable est que la bonne organisation permet de réussir la préparation des étudiants aux différents certificats et à une meilleure insertion dans le milieu professionnel national ayant beaucoup de besoins en compétences bien qualifiées dans le monde des TIC.

\section{c. Certification en développements Informatiques}

D'après l'enseignant responsable :

- L'impact de ces certifications sur la formation : Les modules de certificats passés avec succès sont des preuves réelles de la maîtrise des parties management, architecture, design, accès aux BDD, ajout des fonctionnalités ou utilisation des fonctions et bibliothèques prédéfinies dans la technologie «.NET ».

- L'impact de ces certifications sur l'embauche : dans le marché de l'emploi, les lauréats à la fois diplômés et certifiés ont plus de chances aux bons postes que ceux qui sont uniquement diplômés. Pour l'employeur, le lauréat diplômé et certifié possède parfaitement la maîtrise de la technologie «.NET». Il n'est donc pas nécessaire de le reformer et lui passer des tests sur la technologie. Pour les candidats diplômés uniquement, un passage de test sur la technologie est obligatoire pour pouvoir répondre à certains appels d'offre.

- Le taux de réussite sur chaque module de certificat est presque 95\%. Ce taux est justifié par le fait que lors de la préparation, plusieurs projets sont étudiés pour toucher le maximum d'aspects. Les étudiants qui ne sont pas attentifs ou qui ont des problèmes d'interprétation linguistique ne réussissent pas les tests de certification.

- Les étudiants sont très satisfaits par les ateliers traités et par les certificats passés car pratiquement tous les objectifs de ces ateliers ont été atteints avec succès.

- Il n y a pas de remarque ou réclamation particulières à signaler par l'enseignant, car en réalité, tous les cours et ateliers se sont déroulés dans les bonnes conditions et tous les candidats s'intéressent aux nouvelles technologies de l'information et de la communication en particulier aux technologies «.NET » qui sont des solutions complètes et intégrées.

- Le principal problème rencontré est que pour valider un certificat, il faut que l'étudiant valide toutes les questions $(100 \%)$ ce qui peut être difficile pour les étudiants. Il faudra 
essayer d'envisager prochainement des taux de validation de l'ordre de $80 \%$ ou $85 \%$. l'enseignant se trouve parfois obligé de donner des explications à des étudiants en difficulté.

- Le conseil donné aux étudiants est de tester leurs connaissances et compétences dans des projets industriels et de passer des certificats professionnels «.NET»

- Le conseil donné aux enseignants désirant certifier des étudiants est de bien les préparer aux examens de certificats (de très bons ateliers traitant les questions de l'examen, beaucoup d'explications sur l'architecture «.NET » et beaucoup d'examens blancs).

- La recommandation du responsable est de passer aux étudiants, plus de certificats dans les TIC, c'est très utile et plus rassurant pour un développeur, un ingénieur ou un consultant IT. Les certifications donnent plus d'avantages et plein d'opportunités pour l'avenir.

\section{d. Conclusion}

D'après les avis des enseignants responsable des certifications à l'ENSET, on peut noter les points suivants :

- Un très bon impact des certifications sur la formation et sur l'embauche des étudiants;

- Des très bons taux de réussite et de satisfaction des étudiants dans toutes ces certifications. Ce qui peut justifier l'intérêt que les étudiants portent aux certifications.

- Quelques problèmes de logistique à résoudre pour la bonne conduite de ces certifications (connexion Internet, effectif des étudiants, disponibilité des machines, etc...)

- Incitation des étudiants à passer le maximum de certifications académiques et éventuellement des certifications professionnelles.

\subsection{Avis des lauréats certifiés et non certifiés}

\section{Cas : impact de la certification Cisco sur l'employabilité des étudiants certifiés :}

\section{a. Statistiques et analyse de données}

L'ENSET de Mohammedia a récemment lancé une étude auprès de ses lauréats certifiés pour savoir l'impact de la certification Cisco sur la chance de décrocher un poste d'emploi.

Le questionnaire envoyé par courriers électroniques à plus de 60 lauréats comporte principalement des questions autours $d u$ type de certification académique et/ou professionnelle préparées, type et domaine d'activité actuelle, impact de la certification sur l'embauche et la carrière du lauréat, ... etc.

L'analyse des différentes réponses, nous a permit de conclure ce qui suit :

Tableau 1: Résultats des statistiques : impact de la certification Cisco sur l'employabilité

Total certifiés : 60

\begin{tabular}{|l|c|c|c|c|}
\hline $\begin{array}{c}\text { Certification } \\
\text { CCNA Académique }\end{array}$ & $\begin{array}{c}\text { Nombres } \\
\text { certifiés }\end{array}$ & $\begin{array}{c}\text { \% } \\
\text { Certifiés }\end{array}$ & $\begin{array}{c}\text { Nombre } \\
\text { d'employés }\end{array}$ & $\begin{array}{c}\text { Impact sur } \\
\text { l'emploi }\end{array}$ \\
\hline CCNA Professionnelle & 51 & 85 & 39 & $76 \%$ \\
\hline
\end{tabular}




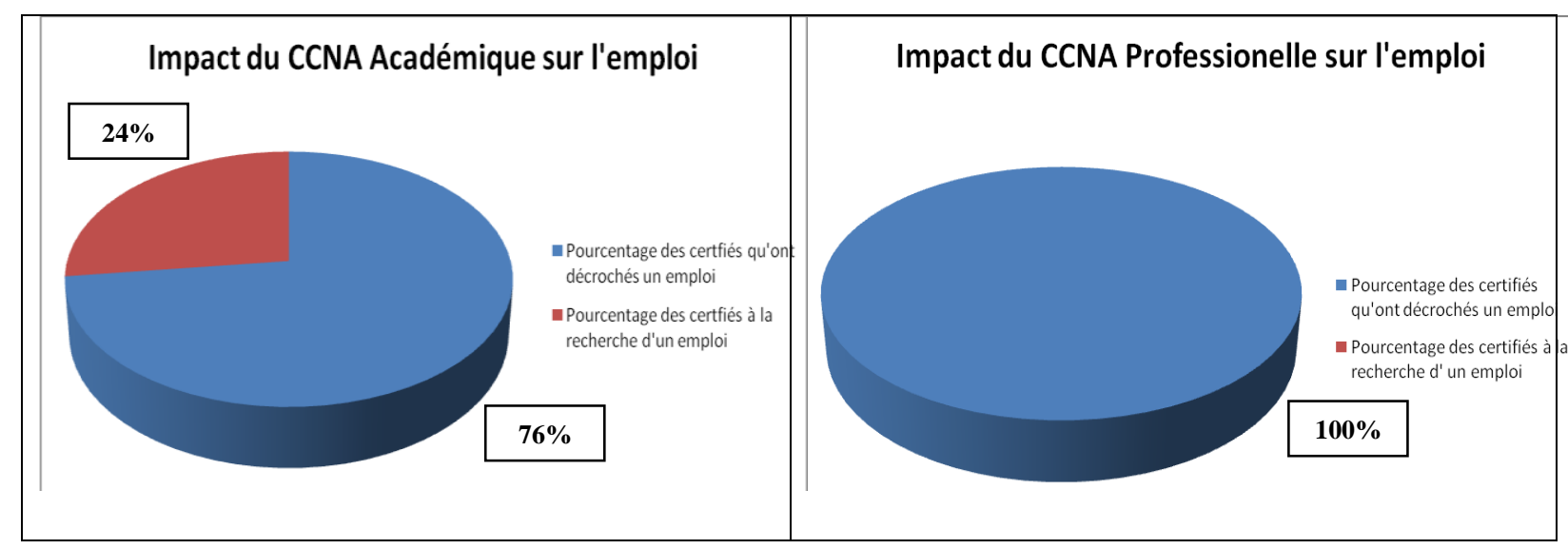

Figure 4 : Impact de la certification CCNA sur l'emploi

b. Conclusion :

D'après les résultats, on remarque que les certifications professionnelles garantissent un emploi à $100 \%$. Les certifications académiques, elles même, offrent des chances de décrocher un emploi à plus de 70\%. D'où l'importance des certifications pour la préparation de carrière.

\section{Proposition d'un modèle de mise en place d'un processus de certification académique dans un établissement d'enseignement supérieur. Cas de la certification Cisco.}

\subsection{Presentation du programme Cisco Networking Academy (NetAcad)}

Lancé en 1997 aux Etats-Unis et en 2001 au Maroc, Le Cisco Networking Academy Program (CNAP) est un programme d'éducation dynamique, à but non lucratif, offrant à des étudiants un enseignement générique sur les technologies des réseaux informatiques, il ne porte pas spécifiquement sur les produits Cisco.

Cisco travaille avec des établissements d'enseignement, des organismes à but non lucratif, des organisations non gouvernementales (ONG) et des centres communautaires qui procurent des salles de classe, des équipements informatiques et des instructeurs qualifiés [19].

Comme illustré sur la figure 5, Le format des cours repose sur un support multimédia qui est mis à la disposition des enseignants et des étudiants à travers un serveur de communauté mondial (www.netacad.com), facilitant ainsi le partage et la transmission des connaissances.

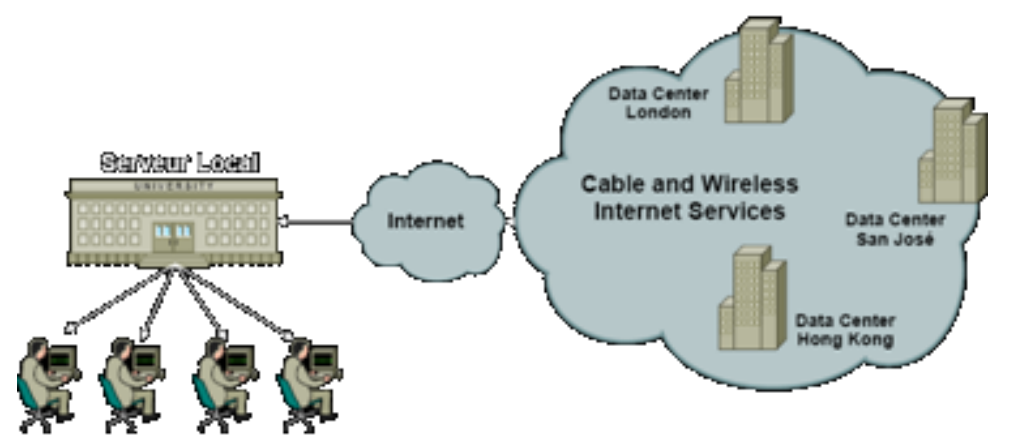

Figure 5 : Support multimédia de cours Cisco

Ce programme permet aux étudiants de se préparer aux certifications reconnues sur le marché pour entamer une carrière dans les technologies de l'information et de la communication (TIC), dans quasiment tous les types d'activité.

Networking Academy a déjà formé plus de quatre millions d'étudiants à ce jour [21]. 
Cisco propose Cinq niveaux de certifications professionnelles [20] (figure 6)

- CCENT : Cisco Certified Entry-level Technician

- CCNA : Cisco Certified Network Associate

- CCNP : Cisco Certified Network Professional

- CCIE : Cisco Certified Internetwork Expert

- Architect : Cisco Architect

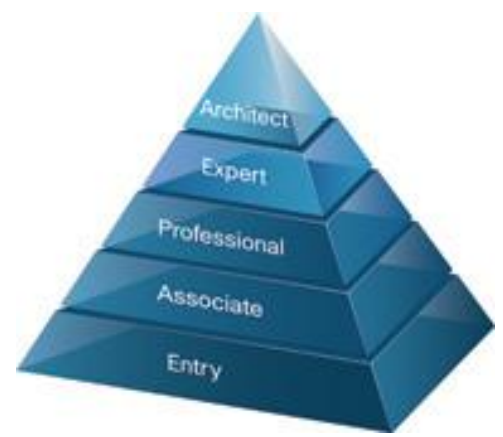

\section{Figure 6 : Niveaux de certifications professionnelles Cisco}

Les cours du programme NetAcad couvrent une large gamme de sujets dans les TIC [21] :

- IT Essentials : Offre un aperçu du matériel et des logiciels ainsi que des systèmes d'exploitation réseau pour préparer les participants aux certifications CompTiA.

- CCNA Discovery : Enseigne la gestion des réseaux domestiques, de petit bureau et d'entreprise et prépare les participants aux certifications Cisco CCENT ${ }^{\mathrm{TM}}$ et CCNA®.

- CCNA Exploration : Enseigne la gestion de réseaux au niveau technologique en offrant un aperçu des protocoles, de la théorie et des technologies avancées afin de préparer les participants à la certification CCNA®.

- CCNA Security : Présente les concepts et les compétences clés liés à la sécurité nécessaires pour installer, dépanner et contrôler les équipements réseau afin de préparer les participants à la certification CCNA Security.

- CCNP : Enseigne les compétences avancées requises pour gérer des infrastructures réseau convergentes de bout en bout pour préparer les participants à la certification Cisco CCNPß .

\subsection{Le modèle d'apprentissage}

Les cours NetAcad sont proposés dans plusieurs langues, selon un modèle d'apprentissage mixte qui combine les cours en salle de classe, les programmes en ligne, les outils interactifs et les exercices pratiques. Les cours sont sanctionnés par des évaluations en ligne. Ce modèle repose sur différentes technologies innovantes [21]:

- Cisco NetSpace: L'environnement de formation en ligne Cisco NetSpace offre les meilleures applications en cloud pour l'enseignement, l'apprentissage et la collaboration. Il propose ainsi une expérience d'apprentissage interactive et attrayante en accord avec les derniers modèles, tendances et meilleures pratiques en matière d'éducation.

- Cisco Packet Tracer : ce puissant outil de visualisation et de simulation permet aux utilisateurs de concevoir, d'élaborer, de dépanner et de tester les réseaux virtuels. Les étudiants et les instructeurs utilisent Packet Tracer pour explorer des concepts techniques et des systèmes de réseau complexes au sein d'un environnement virtuel sécurisé.

- Cisco Passport21 to Entrepreneurship : cette série d'études de cas, de simulations et d'outils interactifs aide à développer des compétences commerciales et financières.

- Cisco Aspire : ce jeu éducatif proposé dans un environnement virtuel permet aux étudiants de relever des défis commerciaux et techniques dans le cadre de projets pour des clients.

- Évaluations en ligne : ces questionnaires portant sur l'ensemble du programme procurent un "feedback" immédiat, interactif et personnalisé aux étudiants.

- Médias sociaux : Facebook, Twitter, LinkedIn et le site NetAcad, une communauté d'instructeurs, permettent aux étudiants et aux instructeurs d'entrer en contact les uns avec les autres d'un bout à l'autre du monde. Cela permet de favoriser la collaboration et de continuer à apprendre au-delà de la salle de classe. 


\subsection{Mise en place de la certification Cisco}

Avant 2012, pour mettre en place la certification académique Cisco, il fallait respecter le modèle hiérarchique à gauche de la figure 7. Depuis 2012, Cisco a mis en place un nouveau modèle organisationnel composé des entités suivantes :

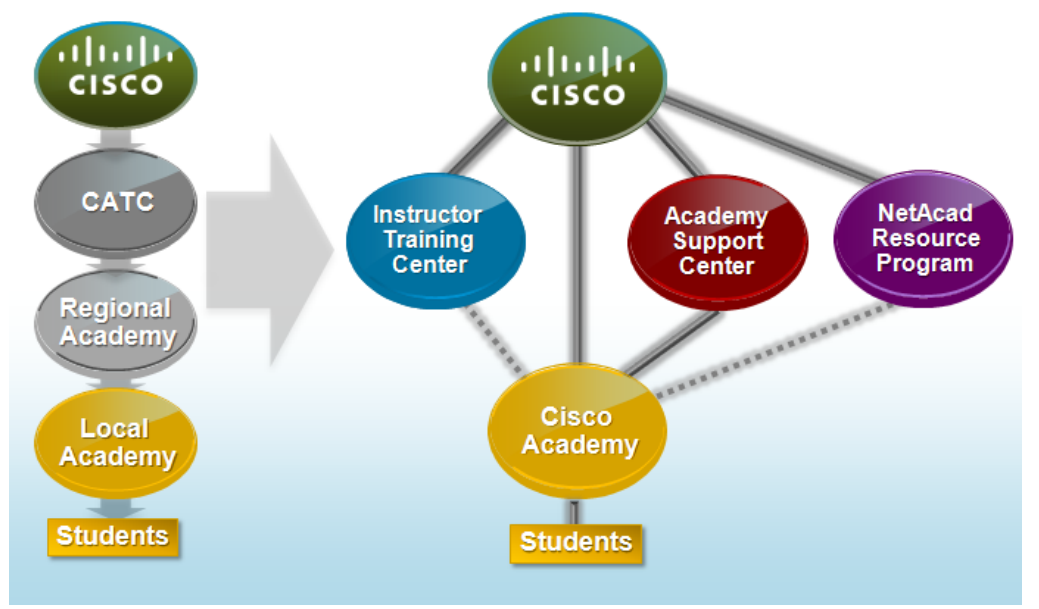

Figure 7 : Migration vers le nouveau modèle organisationnel [22]

- Cisco Academy (CA) : Un établissement éducatif qui Offre aux étudiants le programme de la Cisco Networking Academy,

- Academy Support Center (ASC) une Institution qualifiée pour fournir une assistance de base aux académies et les aide grâce à des activités intégrées et à la localisation de communications critiques,

- Instructeur Training Center (ITC) : Institution qualifiée pour proposer une formation et une assistance technique aux instructeurs pour la première année d'enseignement,

- Netacad Ressource Program : Programme ou service qui encourage et reconnaît les entités qui vont renforcer et valoriser l'écosystème NetAcad. Il Permet aux diverses organisations d'être reconnues pour leurs contributions à l'écosystème de la Networking Academy

\subsection{Expérience de l'ENSET Mohammedia : Projets E-Parité et WIT}

\section{a. Projet E-Parité}

En 2004, le Ministère de 1'Education Nationale, de l'Enseignement Supérieur, de la Formation des Cadres et de la Recherche Scientifique ainsi que le Secrétariat d'Etat auprès du Ministère de 1'Emploi et de la Formation Professionnelle, chargé de la Formation Professionnelle ont signé un accord cadre concernant le projet «Réussir la E-parité dans le domaine des Nouvelles Technologies au Maroc») en partenariat avec l'Agence Américaine pour le Développement International (USAID), le Fonds de Développement des Nations Unies pour la Femme (UNIFEM) et CISCO Systems Inc. (CISCO) [23]. L'objectif de ce projet est de permettre, entre autre, le renforcement du pouvoir économique des femmes marocaines à travers leur participation dans le secteur des TIC et ce en conformité avec les objectifs du plan d'action national E-Maroc. La participation féminine visée est de 40\% [23].

Ce projet vise à renforcer les capacités et les compétences dans le domaine des TIC par la mise en place de 12 «Cisco Networking Academy Programs» (CNAP) et à mettre en lien les étudiant(e)s des CNAP avec le marché du travail.

Pour la mise en place de la certification CCNA, l'ENSET de Mohammedia a réservé à ce programme : deux salles équipées de douze ordinateurs chacune connectés à Internet et dotée d'un vidéo-projecteur et deux formateurs certifiés « instructeurs cisco ». 
Le programme CNAP permet aux étudiants inscrits, l'accès au site d'enseignement de Cisco (www.netacad.com) afin de préparer la certification CCNA académique qui comporte 4 modules. L'étudiant peut choisir le parcours qui lui convient selon le schéma suivant [22]:

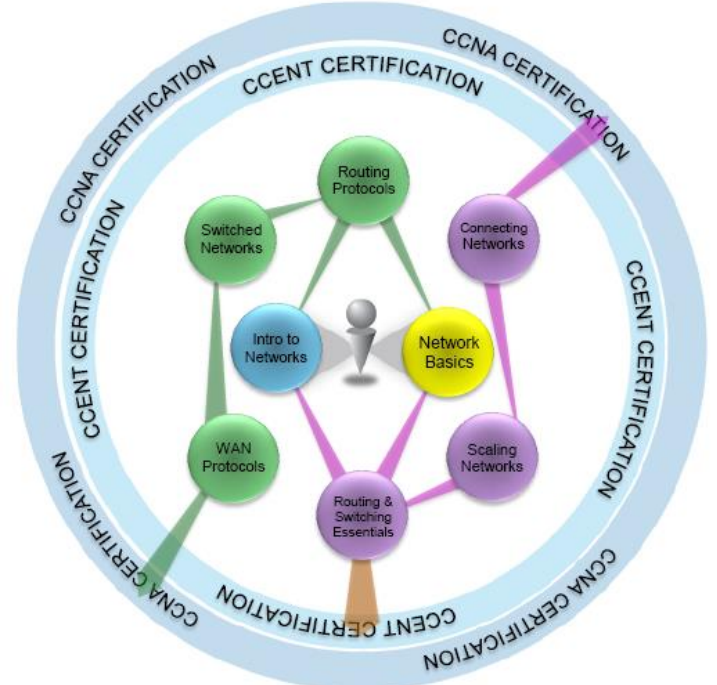

Figure 8 : Parcours à choisir par les étudiants dans la certification Cisco

Un étudiant inscrit dans ce programme s'engage à passer les modalités de contrôle de connaissance sous forme de QCM nécessaire pour valider les modules du programme CNA.

Après validation des 4 modules, l'étudiant reçoit le CCNA académique. Il pourra passer la certification CCNA professionnelle (uniquement l'examen final dont le contenu est identique au CCNA académique) dans un centre d'examen agréé (www. Pearsonvue.com). Les étudiants ne passant par exemple que les modules 1 et 2 se voient également attribuer un papier officiel de Cisco attestant de leur réussite aux modules 1 et 2.

\section{b. Le projet : Women In Technology (WIT)}

Le programme Women In Technology (WIT) octroie des bourses aux candidates originaires des pays du Maroc, de l'Algérie et de la Tunisie, pour s'inscrire au programme CNA afin de préparer le certificat CCNA. Ce programme est sponsorisé par United States Agency for International Development (USAID), administré par Institute of International Education (IIE) (USA) et subventionné par le programme Dot-GOV de Internews Network.

Ce programme de formation a été réalisé en partenariat avec l'ENSET de Mohammedia et l'ENSET de Rabat. Il a permit à des femmes de concevoir, mettre en place et maintenir des réseaux informatiques et préparer les examens des certifications CCNA.

\section{Conclusion générale et perspectives}

A travers cet article nous avons pu montrer que la certification est un processus qui permet de mesurer le niveau de compétence à un moment donné. Il sert comme outil de validation et mise en valeur des compétences et de connaissances acquises dans une formation.

Nous avons aussi étudié la différence entre la certification professionnelle et la certification académique et nous avons pu montrer à travers des statistiques, que les certifications donnent plus de chances aux lauréats pour décrocher un bon emploi et dans de grandes structures.

Les enseignants responsables des certifications pensent que celles-ci ont un très bon impact sur la formation et sur l'embauche des étudiants. Ils ont souligné le grand intérêt porté par les étudiants à toutes les certifications ce qui justifie les taux de réussite. Ils ont conseillé à l'incitation de tous les étudiants à passer un maximum de certifications académiques et professionnelles. 
Enfin on a proposé un modèle de mise en place d'un processus de certification académique dans un établissement de l'enseignement supérieur, cas de la certification Cisco, en s'inspirant de l'expérience de l'ENSET de Mohammedia dans le domaine.

Nous souhaitons par la suite approfondir nos études sur les certifications académiques et professionnelles dans d'autres domaines techniques et chercher leurs impacts sur l'amélioration de la formation et l'insertion des lauréats dans le monde socio-économique

\section{Références:}

[1] Article collectif de l'association REBIS, Eléments problématiques pour l'ingénierie en formation d'adulte, 1996.

[2] Fernandez, J., Réussir une activité de formation, Montréal, p 21, 1988.

[3] Philippe Perrenoud, A., La qualité d'une formation se joue d'abord dans sa conception, vol. 11, pp 4, 1998.

[4] J.Coquerel, De la formation à la compétence, Forum Alger, Alger, 2004.

[5] CREFOR HAUTE- NORMANDIE, Le paysage français des certifications à finalité professionnelle, Éclairage, Rouen, 2009.

[6] A. Vinokur, A., La qualité de la mesure de la qualité dans l'enseignement supérieure : essai d'analyse économique, Éducation et société, Paris, 2006.

[7] J.Teissier et J.Rose, A., La certification nouvel instrument de la relation formation emploi, RELIEF 16 Céreq, France, 2006.

[8] F.Maillard et J Rose, A., Les diplômes de l'éducation nationale dans l'univers des certifications professionnelles, RELIEF 20 Céreq, France, 2007.

[9] J.Dahan, La formation : le chemin pour la construction d'un métier, Empan, N 72, 2008.

[10] S.Dumartin, A. Formation-emploi : quelle adéquation?, Économie et statistique, numéro 3, 1997.

[11] B.Conter et C.Maroy, Le développement de la formation professionnelle continue en Belgique francophone, GIRSEF, numéro 2, 1999.

[12] J.Castel et A.Troubat, Les compétences comme ROI de la formation, meta4, 2004.

[13] L.Lahlou, La réforme de l'Université marocaine entre idéal organisationnel et réalité pratique, Colloque international, Paris, 2009.

[14] Y.Marcyan, La professionnalisation des diplômes universitaires : la gouvernance des formations en question, Thèse, Nancy, 2010.

[15] M.Otter, J.Sidi et L.Hanaud, Guide des certifications SI Comparatif, analyse et tendances ITIL, CobiT, ISO 27001.

[16] Site Officiel d'Oracle, http://education.oracle.com/

[17] Site officiel Cisco http://www.cisco.com/web/FR/events/training/certification/index.html

[18] Site officiel de Microsoft, http://www.microsoft.com/france/education/

[19] globalnewsroom.cisco.com

[20] www.cisco.com/web/learning/certifications/retired/ccip_retired.html

[21] www.netacad.com/

[22] honim.typepad.com/biasc/2013/05/ccna-50-overview.html

[23] www.lemag.ma/Reussir-la-E-parite-dans-le-domaine-des-NTIC-au-Maroc a3573.html 